\title{
Women and Management of Menopause at Old Age for Graceful Living
}

\author{
Florence A. Undiyaundeye (Ph.D) \\ Dept. of Early Childhood Care Development Education Federal College of EducationObudu, Cross River State \\ Nigeria
}

\begin{abstract}
This paper intends to deal with menopause and how the stressful effect can be managed for a graceful old age. Menopause represents a part of adjustments a woman goes through in midlife. Even though this period ends fertility, it requires the women to stay emotionally and physically healthy. The knowledge on menopause assist counsellors, clinicians, and all those who handle women issues to plan and provide comprehensive counseling to women about managing the stress of menopause. Menopause is a natural phenomenon which occurs in all women when their finite number of ovaries follicles are depleted. It starts with premenopausal transition which is a period of change leading up to the last period. The relationship between menopause and the development of associated conditions is sometimes difficult to differentiate from age related morbidity but behaves clearer if education and consequences are handled by an emotional manager. Menopause begins silently from 40 years when estrogen level starts declining and the progesterone causes menstruation to become irregular leading to physical changes like reduction in pregnancy, reduce sex drive and mood change. All these symptoms and others could cause some distress in the victim. An emotional manager who could be a counsellor or a clinician may prescribe a healthy dieting, hormone replacement therapy and complimentary therapy. All these does not only provide the body with the required nutrients but also help to balance hormones, improve mood and brain chemistry. All these leads to graceful living. To this end, counseling women about hormonal changes that occur at menopause, its effect on these change and ways of preventing or treating conditions associated with menopauseis needed for graceful living.
\end{abstract}

Keywords: Women, Menopause, Old age, Graceful living, Management.

\section{Introduction}

Menopause is a natural phenomenon or a biological process which occurs in all women when menstruation and fertility ends permanently. Even though it ends fertility, the woman can stay healthy, vital and sexual in a graceful old age. It happens when the woman finite number of ovaries follicle are depleted as estrogen and progesterone hormone level fall. Panay (2013) posits that menstruation becomes erratic and eventually stops and that menopause is a retrospective diagnosis of the time when menstruation permanently ceases.

Women reaction to menopause varies as some mourn the end of youth and fertility while others welcome it as a time of freedom and new opportunities. Either way, menopause is a universal rite of passage for women, marking significant physical and emotional changes which can require some adjustments for ageing positively. Schairer, Lubin and Troisi (2000) is of the view that technically, menopause refers to the time when women menstruation ceases and a gradual process of reproductive ageing including perimenopause and postmenopause for most women the process of menopause begins silently between the ages of 40 and 58 with an average of 51 years. A few women reach menopause in their thirties and this is called premature while another small number don't reach menopause until they are 60 in age.

The most likely predictor of how women experience menopause is hereditary that is how one's mother or grandmother fared could be a point of inference in the current. Menopausal transition and its endocrine correlates suggests that division of the menopausal transition into discrete stages enhances our ability to understand its relationship to health outcomes. A population based study of women in midlife by Obstetand Gynaecol (2008) suggest three menopausal transition stages namely: early, middle and late. In the early stage, women have regular of menstrual flow (often lighter, but it may be heavier and involve flooding or spotting). During middle stage women's menstrual cycle are irregular (with variability of six days or more between consecutive cycles) but women do not notice skipping periods meaning the interval between their cycles is double or more of the length of their usual menstrual cycle.

\section{Symptoms during Menopausal Transition}

Most women experience some symptoms as they go through the change of life cycle into menopause. By being aware of the changes ahead and meeting them heed on with healthy diet, it can ensure that the 
transitional time involves some change for the better (Nawaz \& Katz 2009; Undiyaundeye 2013). Perimenopause, the period preceding menopause is often more dramatic than menopause itself. During this preliminary phase, hormone levels fluctuate widely causing a variety of symptoms including:

- Hot flashes: This is experienced by 75 - 80 percent of all women which range from a strong blush to profuse sweating with intense heat, usually starting at the head and the neck.

- Menstrual cycle change: Menses become either heavier or lighter, occur more or less frequently, last longer or shorter in duration.

- More changes: You may find yourself feeling more irritable, teary, emotionally detached or worried than usual or feel a vague sense of anxiety without a particular cause. Many women experience poor motivation and a general sense of fatigue.

- Change in appetite: Women may experience food cravings especially during the second half of menstrual cycle. These could be increased appetite or suffer from nausea.

- Sleep disturbances: disrupted sleep patterns including difficulty falling asleep or waking in the middle of the night and not being able to go back to sleep. Sleep problems can lead to feelings of depression.

- Memory changes: A feeling of if things are forgotten more easily. This may be due to lack of sleep or the fact that there is a decreased estrogen levels and reduced the hundreds of estrogen receptors on the brain, thus affecting brain function.

- Urinary symptoms: Women may discover that there is frequent urination, slight leakage on sneezing, coughing or laughing. It could also be a common increase in urinary tract infection due to changes in the normal bacteria in the vagina.

- Sexual changes: Due to lowered estrogen levels the women's libido at this stage decreases. Dryness in the vagina the women may feel pain or discomfort during intercourse or even light spotting after sex because the cervix's lining is more fragile and thin.

- $\quad$ Skin sensitivity: Some women experience cranking skin, a tingling dry or even burning sensation.

- Joint and muscle aches and pain

- Digestive disturbances: Heartburn is a common complaint

- Health jitters: The feeling of a pounding or racing heart can be scary. This pounding is harmless to the body and may be accompanied by shortness of breath and hot flashes.

- Ovarian growth: Women may suffer from the growth of being ovarian cysts. Consulting a doctor to verify if it is not serious would ease the anxiety.

\section{Strategies for Managing Menopause for a Graceful Living}

In managing menopausal symptoms of women experiencing midlife symptoms of premenopausal and menopausal experiences within this stage; the counsellor and clinicians must ascertain the root causes of a particular dysfunction through leading questions to curb the situation as observed by (Utian, Archer \&Bechmann, 2008; Philip, 2000 and Undiyaundeye, 2005):

- It is pertinent to organize and plan one's life style to the extent of knowing what works for you and what does not. Do things that one is comfortable with and worry less about extra things.

- Hot flashes are the most common symptoms of menopause to some degrees. Regular aerobic exercise can help decrease hot flashes and also try to pinpoint what triggers the hot flashes e.g. hot drink, spicy food, alcohol, avoid caffeine by laughing hot weather. Either avoid or modify the trigger if possible.

- Vaginal dryness/dyspareumia - Vaginal lubricants/moisturizers can make intercourse more comfortable and regular. Sex may help the vagina maintain its elasticity.

- Laughing helps relieve stress that means it should be in practice even when the victim is not in need. Laughing is a powerful antidote to stress, pain and conflict. Nothing works faster or more dependably to bring one's mind and body back into balance than a good laugh. Humour lightens, inspires hope, connect one to others and keep one grounded focused and alert. Surrounding oneself with family, love ones and friends triggers the body natural feel-good chemicals, promotes the release of endorphins which generate overall sense of well being and can temporarily relieve pain.

- Learn how to incorporate soy foods into your diet. Soy food contains isoflavones (plant hormones) that act like a weak form of estrogens in the body.

- Bring on the beans (legumes). They contain package of fiber, protein, calcium, folic acid and plytoestrogens which help with blood sugar control. Add canned legumes to salads, pastas, soups, and stews each week.

- Good sources of zinc like lean meat, seafood, eggs and milk keeps the body immune system in tip-top shape.

- $\quad$ Boost the baron needs by eating a variety of fruits and vegetables regularly

- Avoid saturated and trans fats which tend to raise blood cholesterol levels and increase risk for heart disease in post-menopausal women. 
- Watch for hidden sugar by lurking in your foods. Too much sugar can cause blood sugar to spike which estimates the pancreas to release more of the hormone insulin that means the consumption of soft drinks, syrups, jams, table sugar, candy, sweetened yogurt and sugary breakfast cereals should be limited

- $\quad$ Feast on flax in the diet is the safest way to help with hormonal balance during menopause.

Going through the strategies as mentioned, it helps in managing and getting through the physical and conditional changes that can accompany menopause along with helping the women embrace a graceful living.

\section{Conclusion}

Managing menopause is a part of a number of adjustments that women face at midlife stage. Though many women talk about menopause as if it were a curse, it can be a very enjoyable time if intervention to curb the negative feelings is prescribed by expertise strategies. Strategies for preventing the diseases associated with advancing in age can be multi-faceted and include dietary exercise and possibly lifestyle changes as well as pharmacologic interventions. Counseling should consider the broad range of pharmacologic and nonpharmacologic interventions that can address health considerations under discussion at the time of menopause. Counseling should also include the strength of the supporting scientific evidence for each of the interventions.

\section{References}

[1]. British Menopause Society and Bileman's Health Concern Recommendations on Hormone Replacement Therapy, Panay N. (May, 2013)

[2]. British Menopause Society and Women's Health Concern Recommendations on Hormone Replacement Therapy, Panay L.A (May, 2013)

[3]. Nawaz, H and Katz D.L. American College of Preventive Cardiollogy for Women: Perimenopausal and Postmenopausal Hormone Replacement Therapy. AMJ Prev Med 2009; 17 (3): 250 - 254.

[4]. Philips, N.A. (2000). FemaleSexual Dysfunction: Evaluation and Treatment. New Zealand, University of Otego Wellington.

[5]. Schairer, C. Lubin J., Troisi R. Menopausal Estrogen - Progestin Replacement Therapy and Breast Cancer Risk. JAMA 2000; 283 (4): $485-491$

[6]. The Canadian Consensus Conference on Menopause and Osteoporosis. Part 1: Consensus Statement Obstet J.S and Gynaecol, C. 2013: $20(13): 501-612$

[7]. Undiyaundeye, F.A (2005). Family Counseling and Sexual Adjustment in Marriage. In Ugbe (Eds) (2005). The Basics of Guidance and CounsellingObudu. Austin Printers and Publishers.

[8]. Undiyaundeye F.A (2013). Menopause in Women. Implication for Counselling. Global Advanced Research:Journal of Educational Research and Review vol. 2 (1) pp $41-45$.

[9]. Utian W.H, Archer D.F, Bechmann, G.A; Estrogen and Progestogen Use in Post menopausal Women. July 2008 Position Statement of the North American Menopause Society. 15 (4) pt $1584-602$. 